\title{
Melamine Analysis in Liquid Milk by Simple and Robust Neural Network Based Method
}

\author{
Sergey V. Smirnov
}

$R \& D$ department, Unimilk Co., Moscow region, Russia

\begin{abstract}
Melamine (2,4,6-triamino-1,3,5-triazine) is a nitrogen-rich chemical implicated in the pet and human food recalls and in the global food safety scares involving milk products. Due to the serious health concerns associated with melamine consumption and the extensive scope of affected products, rapid and sensitive methods to detect melamine's presence are essential. We propose the use of spectroscopy data - produced by near-infrared (near-IR/NIR) and mid-infrared (mid-IR/MIR) spectroscopies, in particular - for melamine detection in complex dairy matrixes. It was found that infrared spectroscopy is an effective tool to detect melamine in liquid milk. The limit of detection (LOD) below $1 \mathrm{ppm}(0.76 \pm 0.11 \mathrm{ppm})$ can be reached if a correct spectrum pre-processing (pre-treatment) technique and a correct multivariate (MDA) algorithm: partial least squares regression (PLS), polynomial PLS (Poly-PLS), or artificial neural network (ANN) - is used for spectrum analysis. The relationship between MIR/NIR spectrum of milk product and melamine content is nonlinear. So, non-linear regression methods are needed to correctly predict the triazine-derivative content. It can be concluded that mid- and near-infrared spectroscopy can be regarded as a quick, sensitive, robust, and lowcost method for liquid milk analysis. The technique can be applied for the automation of milk analysis.
\end{abstract}

Index Terms: food; liquid milk; partial least squares regression (PLS); artificial neural network (ANN)

(C) 2011 Published by MECS Publisher. Selection and/or peer review under responsibility of the International Conference on E-Business System and Education Technology

\section{Introduction}

Melamine (2,4,6-triamino-1,3,5-triazine) is a nitrogen-rich chemical implicated in the pet and human food recalls in 2007 [1,2] and in the global food safety scares in 2008 involving milk products [3-5]. In those food safety incidents, melamine was intentionally added to foods and animal feed to boost the protein content [6,7]. A driving force for the adulteration of a food product with melamine is that its high nitrogen content, which increases the apparent protein content measured by standard protein analysis tests, such as Kjeldahl or Dumas ones [5]. Note that the Kjeldahl analytical method is a method for the quantitative determination of nitrogen only (not true protein content) [8]. 
In late 2008, trace amount of melamine were detected in US-made infant formula products $[9,10]$. The recalls involving pet food and milk products, contaminated with melamine, have created a widespread food safety scare $[5,8]$. Today melamine contamination has been reported in a variety of food products such as milk (liquid or powder), infant formula, frozen yogurt, pet food, biscuits, candy, and coffee drinks [11].

The 2008 incident of contamination of milk with melamine in China likely caused 300,000 cases of renal complications in children, and at least 6 child deaths, directly resulting from consumption of tainted product [5,12]. Toxic effects associated with melamine consumption occur only following high doses [13]. The oral 50\% lethal dose (LD50) has been reported as approximately $3 \mathrm{~g} \mathrm{~kg}-1$ of body weight [14]. It is thought that simultaneous ingestion of melamine and one of its analogues, cyanuric acid, may result in the formation of crystals in the kidney [13], as was the case in the pet food incident [15].

We propose the use of spectroscopy data - produced by near infrared (near-IR or NIR) and medium infrared (mid-IR or MIR) spectroscopies, in particular - for melamine detection in complex matrixes.

Near infrared spectroscopy in combination with MDA methods has been applied to melamine detection in a number of analytical studies [16-35].

In 2009, Mauer et al. [8] evaluated near- and mid-infrared spectroscopy methods (NIR, FTIR-ATR, FTIRDRIFT) for the detection and quantification of melamine in infant formula powder. Partial least-squares (PLS) models were established for correlating spectral data to melamine concentration: R2 $>0.99$, RMSECV $\leq 0.9$, and residual prediction deviation (RPD) above 12. Factorization analysis of spectra was able to differentiate unadulterated infant formula powder from samples containing $1 \mathrm{ppm}$ melamine with no misclassifications, a confidence level of $99.99 \%$, and selectivity > 2. It was stated that NIR and MIR methods enable rapid detection of $1 \mathrm{ppm}$ melamine in infant formula powder [8].

$\mathrm{Lu}$ et al. [36] established a novel and rapid method for detecting pure melamine in milk powder using near infrared (NIR) spectroscopy based on least squares-support vector machine (LS-SVM). Partial least square discriminant analysis (PLS-DA) was used for the extraction of principal components (PCs). The scores of the first two PCs have been applied as inputs to LS-SVM. Compared to PLS-DA, the performance of LS-SVM was better, with higher classification accuracy, both $100 \%$ for the training and testing set. The detection limit was lower than $1 \mathrm{ppm}$. Based on the results, it was concluded that NIR spectroscopy combined with LS-SVM could be used as a rapid and accurate method for detecting pure melamine in milk powder.

In this paper, we try to reach two goals: (i) to establish a quick, sensitive (LOD $<1 \mathrm{ppm}$ ), reliable, and robust method for melamine detection in liquid milk based on NIR and MIR spectroscopy methods; (ii) to compare different multivariate calibration models (PLS, OPLS, ANN, SVM, etc.) on the large melamine data sets (>660 samples each) to find the best candidate for industrial analytical application. A large calibration range of melamine concentration (0.11-2000 ppm) was used to build a model that is capable of dealing with both, almost melamine free and largely contaminated, dairy samples. Notably, the artificial neural networks (ANNs) have never been used to predict melamine content in milk products from vibrational spectral data [36-43].

\section{Experimental}

\subsection{Materials}

Six hundred sixty (660) liquid milk samples were prepared for MIR/NIR analysis from standard (noncontaminated) products, supplied by Unimilk Joint Stock Co. and purchased in a local store. Such a huge amount of samples allows one to estimate the efficiency of the model at the "sample set limit" - see the "basis set limit" (BSL) or "complete basis set" (CBS) in quantum chemistry [43-46], e.g. in focal point analysis (FPA) schemes $[43,47,48]$. The initial samples (12) of liquid milk were checked for the absence of melamine contamination using standard HPLC-based method [49]. 


\subsection{Methods}

The near infrared (NIR) spectra were acquired with a MPA Multi Purpose FT-NIR Analyser (Bruker, Germany). The spectra were acquired at room temperature $\left(20-23{ }^{\circ} \mathrm{C}\right)$. The NIR spectrometer was calibrated with benzene (C6H6) and cyclohexane (c-C6H12) at least twice per day to minimise the influence of variable laboratory conditions. The spectral range between 9000-4500 cm-1 (1110-2500 nm) was scanned with a resolution of $8 \mathrm{~cm}-1$ (Table 2). Thirty-two (32) scans were averaged for each sample spectrum. A background spectrum (32 scans) was measured every $45 \mathrm{~min}$. A photometric accuracy of $\sim 0.07 \%$ was obtained [28,29]. A cylindrical glass cell was used throughout the study. Approximately $1 \mathrm{~mL}$ of sample was needed for each NIR measurement. NIR spectrum collection was repeated 5 times with cell rotation inside the spectrometer to minimise interferences from the cell or glass defects. The measurement of one sample took less than 3 min. The averaged and background-corrected spectrum was used for subsequent data pre-processing [37-39]. See [34,37$60]$ for details.

The IR spectroscopy method was used for determination of the melanine content in milk products. An analysis was performed using a Tensor 27 FT-IR Spectrometer (Bruker, Germany). A MIRacle ATR add-on device (Bruker, Germany) with a ZnSe crystal (1-reflexion; $1.8 \mathrm{~mm}$ ) was used for the analysis of dairy products $[33,35]$. A spectral range between 500 and $4000 \mathrm{~cm}-1$ was scanned with a spectral resolution of $2 \mathrm{~cm}-1$ (Table 2). Thirty-two (32) scans were averaged for each spectrum. The measurement was repeated at least 5 times for each liquid or solid sample. See [33] and Table 2 for details.

Data pre-processing was done according to [37]. Briefly, prior to calibration model building, various widely used pre-processing techniques were applied to the data. Nine (9) data pre-treatment methods were tested.

See $[38,39]$ for detailed discussions of different multivariate methods and algorithms: partial least squares regression / projection to latent structures (PLS), orthogonal projection to latent structures (OPLS/O-PLS), polynomial partial least squares regression (Poly-PLS), and artificial neural networks (ANNs). Spline-PLS method has not been applied due to its relatively high computational cost (long optimization procedure) and almost no superiority in accuracy over simpler Poly-PLS method [38]. The same can be said about principal component regression (PCR) in comparison with PLS method [38].

Here we will try to understand the extent to which ANN-based techniques can substitute linear (e.g., PLS) ones in real-world (industrial) applications.

\section{Results and discussion}

\subsection{Methods optimization}

Cross-validation error was minimized in each case. It is interesting to note that the same degree of polynomial (3) of the final models was found for both methods. The quality of Poly-PLS method was found to be less dependent on the choice of $n$. Other chemometric algorithms (PLS, OPLS, ANN, etc.) were optimized in a similar manner [38].

\subsection{Sample set separation}

It is a hard task to make a general calibration model for a really wide range of melamine content in dairy products, like we have in our sample $(0-2000 \mathrm{ppm})$. If the whole data set is used for calibration model building, the final model will be biased towards the accurate prediction of samples with high melamine content, due to the minimization of mean squared error (MSE) in most multivariate algorithms (10002 >> 1002) [50]. So, the price 
will be low model accuracy for diary samples with low melamine content and, as a consequence, high limit of detection (LOD). That is unacceptable from a point of view of food product quality control [1].

To solve this problem the samples were separated into two subranges, named 'low' and 'high', with melamine content of below $17.3 \mathrm{ppm}(14.6 \mathrm{ppm})$ and between 17.3 and $2000 \mathrm{ppm}$ (14.6-2000 ppm) for infant formula (milk powder / liquid milk). Separate calibration models were built for each range. See Table 1 for details. It was checked that the accuracy of, both, 'low' and 'high' multivariate models in region of the border values (17.3/14.6 ppm) is not worse than elsewhere.

\subsection{MDA methods comparison: ANN-based regression}

The results of multivariate methods comparison for dairy products with low melamine content are discussed below. For both spectroscopic ranges and all milk products the same trend is well-seen: linear calibration methods (PLS and OPLS) show much larger prediction error (RMSEP), exceeding $1 \mathrm{ppm}$. The average error of PLS/OPLS methods is $1.31 \pm 0.07 \mathrm{ppm}(\sigma)$, while the error of Poly-PLS and ANN methods is almost 5 times smaller $(0.28 \pm 0.05)$.

So, one can state that there is definitely a non-linear dependence between infrared spectrum of milk sample and the melamine content even in a low concentration range [1,36-39]. This non-linearity can be corrected by rather simple Poly-PLS algorithm [37].

Almost identical results are observed for all dairy products in both frequency ranges, with the lowest prediction errors of $0.25 \pm 0.04 \mathrm{ppm}$ for SVM-based methods. ANN results of almost the same quality can also be obtained. There is a small superiority of SVR method over LS-SVM one, but the difference is negligible - below the reference method / preparation accuracy $(0.01 \pm 0.01 \mathrm{ppm})$. So, both methods are equally effective and lead to LOD of $0.76 \pm 0.11 \mathrm{ppm}[1]$.

It is clear that linear models are still not accurate. But a different trend is observed in the high-concentration case: the Poly-PLS model, being accurate for low-content region (Figure 3), is not accurate for higher concentrations of the target substance. The ration between the RMSEP of Poly-PLS and the lowest RMSEP has increased from $1.3 \pm 0.1$ to $2.3 \pm 0.6$.

So, for higher melamine concentration the polynomial modification of PLS method is not able to fully account for spectral non-linearity. This can be due to higher influence of intermolecular interactions between melamine and matrix molecules, which are able to slightly shift vibrational frequencies and modify spectral intensities [28-30,56-58].

ANN, SVR, and LS-SVM methods are able to solve this problem. All of them are able to produce accurate results with RMSEP of $6.1 \pm 0.9 \mathrm{ppm}$.

Note that for high melamine content NIR spectroscopy shows superiority over MIR one [22,23,36,59].

Currently, the FDA uses a liquid chromatography-triple-quadrupole tandem mass spectrometry (LC-MS/MS) method to detect residues of melamine in dry infant formula [60]. Although this method provides limits of detection as low as $0.25 \mathrm{ppm}$, the sample preparation and cleanup procedures are time-consuming and laborintensive.

The spectroscopic method for melamine detection based on mid- and near-infrared spectroscopy provides a much quicker and low-cost method procedure with a detection limit of just $0.76 \pm 0.11 \mathrm{ppm}$. Even though this value is by a factor of three larger than LC one, it is still below $1 \mathrm{ppm}$ [1]. That means that the method can be applied in practice to detect adulteration of dairy products by melamine [1].

The influence of matrix is rather limited and different milk products can be analysed with equal efficiency. So, NIR spectroscopy is robust enough for real-world applications. Extra research is needed to clarify the possibility of melamine detection by infrared spectroscopy in other food products.

The presented results can be compared with NIR spectroscopy of petroleum products (e.g., gasoline), as reported by Balabin et al. in 2007 [38]. Note that the hierarchy of the multivariate methods was different in that case: Poly-PLS method was less effective than ANN one. Unfortunately, no information on SVM-based 
multivariate methods was provided [38,39]. It seems to be that the smaller spectral non-linearity was observed in a hydrocarbon mixture.

Unfortunately, up to date not many papers compare several chemometric algorithms to find the most appropriate one for this or that task. So, it is hard to make any general comparison of dairy products with other chemical systems to understand the reasons of good or bad behavior. We can just state that ANN models were found to be the most accurate for the melamine calibration task.

However further work is needed to enlarge the sample banks and the spectral libraries to get a real insight into the chemical reasons of success or failure of the NIR/MIR+MDA strategy.

\subsection{Methods comparison. ANN superiority}

Here we confirm the results of [36] for milk powder and extend them to other dairy products. Both infraredbased methods (MIR and NIR) can be successfully applied to detect melamine in dairy products (liquid milk) with a limit of detection below $1 \mathrm{ppm}$. The simplicity of the sample preparation procedure and recent progress in hand-held near infrared devices makes the proposed technique promising for real-world applications.

At least preliminary food quality control can be done by IR methods with subsequent confirmation of sample contamination by chromatographic method. This will greatly decrease the number of samples, one need to analyse, to ensure food quality and safety. The same technique is applied today in petroleum industry for online analysis of products of petroleum refining and petrochemicals [20,22,23].

The possibility of online (real-time) quality control by NIR spectroscopy is also an interesting perspective for practical implementation of the method proposed. A correct signal pre-processing procedure makes the sample collection unnecessary.

\section{Conclusion}

The following conclusions can be drawn:

(1) Infrared spectroscopy (MIR or NIR) when combined with artificial neural networks (ANNs) is an effective tool to detect melamine in dairy products, such as liquid milk.

(2) The limit of detection (LOD) below $1 \mathrm{ppm}$ can be reached if a correct multivariate algorithm (ANN) is used for spectrum analysis $(\mathrm{LOD}=0.76 \pm 0.11 \mathrm{ppm})$.

(3) Neural network based method for melamine analysis in liquid milk based on spectroscopy data was successfully developed and tested [37-61].

\section{Acknowledgment}

S.V.S. thanks Balabin R.M. (ETH Zurich, Switzerland) for his help with NIR/MIR experiments and paper preparation.

\section{References}

[1] M. Lin, Front. Chem. Eng. China 3 (2009) 427-435.

[2] B. Liu, M. Lin, H. Li, Sens. \& Instrumen. Food Qual. 4 (2010) 13-19.

[3] R. Z. Syunyaev, R. M. Balabin, J. Dispersion Science Tech. 29, 1505 (2008).

[4] H. Xin, R. Stone, Science 322 (2008) 1310-1311.

[5] E.Y.Y. Chan, S.M. Griffiths, C.W. Chan, Lancet 372 (2008), 1444-1445.

[6] K. Burns, S. Kahler, J. Am. Vet. Med. Assoc. 230 (2007) 1784-1785.

[7] S. Wong, M. Chiu, H.K. J. Paediatr. (New Series) 13 (2008) 230-234. 
[8] L. J. Mauer, A. A. Chernyshova, A. Hiatt, A. Deeding and R. Davis, J. Agric. Food Chem. 57 (2009) 39743980.

[9] R. Z. Syunyaev, R. M. Balabin, J. Dispersion Science Tech. 28, 419 (2007).

[10] R. Z. Syunyaev, R. M. Balabin, I. S. Akhatov, J. O. Safieva, Energy Fuels 23, 1230 (2009).

[11] World Health Organization. Melamine contamination event, China, 2008; retrieved Oct, 2009, Grand jury indicts companies for melamine contamination of pet food. JAVMA 2008, 232, 824.

[12] U.S. Food and Drug Administration. Interim safety and risk assessment of melamine and its analogues in foods for humans; http://www.cfsan.fda.gov/ dms/melamra3.html.

[13] Melamine; MSDS 11295; in Alfa Aesar, Ward Hill, MA; http://www.vwrsp.com/msds/10/AAA/AAA11295-0C.pdf.

[14] R. L. Dobson, et al. Toxicol. Sci. 106 (2008) 251-262.

[15] L. He, Y. Liu, M. Lin, J. Awika, D. R. Ledoux, H. Li, A. Mustapha, Sensing and Instrumentation for Food Quality and Safety 2 (2008) 66-71.

[16] D. N. Heller, C. B. Nochetto, Rapid Communications in Mass Spectrometry 22 (2008) 3624-3632.

[17] G. Huang, Z. Ouyang, R. G. Cooks, Chem. Commu. 5 (2009) 556-558.

[18] C. M. Karbiwnyk, et al. Analytica Chimica Acta 637 (2009) 101-111.

[19] R. M. Balabin, R. Z. Safieva, E. I. Lomakina, Anal. Chim. Acta 671 (2010) 27-35.

[20] S.B. Kim, C. Temiyasathit, K. Bensalah, A. Tuncel, J. Cadeddu, W. Kabbani, A.V. Mathker and H. Liu, Expert Sys. Appl. 37 (2010) 3863-3869.

[21] R.M. Balabin and R.Z. Safieva, J. Near Infrared Spec. 15 (2007) 343-349.

[22] R.M. Balabin and R.Z. Safieva, Fuel 87 (2008) 2745-2752.

[23] A. Jiye, J. Trygg, J. Gullberg, A.I. Johansson, P. Jonsson, H. Antti, S.L. Marklund and T. Moritz, Anal. Chem. 77 (2005) 8086-8094.

[24] M.R. Monteiroa, A.R.P. Ambrozin, M.S. Santos, E.F. Boffo, E.R. Pereira-Filho, L.M. Lião and A.G.

Ferreira, Talanta 78 (2009) 660-664.

[25] J. Workman, Jr., M. Koch, B. Lavine and R. Chrisman, Anal. Chem. 81 (2009) 4623-4643.

[26] P. Baptista, P. Felizardo, J.C. Menezes and M.J.N. Correia, Talanta 77 (2008) 144-151.

[27] R.M. Balabin, J. Phys. Chem. A 113 (2009) 4910-4918.

[28] R.M. Balabin, J. Phys. Chem. A 113 (2009) 1012-1019.

[29] R.M. Balabin, J. Phys. Chem. Lett. 1 (2010) 20-23.

[30] J.M. Hollas, Modern Spectroscopy (4th ed.), Wiley (2004).

[31] R.M. Balabin, R.Z. Syunyaev and S.A. Karpov, Fuel 86 (2007) 323-327.

[32] R.M. Balabin, R.Z. Syunyaev and S.A. Karpov, Energy Fuels 21 (2007) 2460-2465.

[33] F.C.C. Oliveira, C.R.R. Brandao, H.F. Ramalho, L.A.F. Costa, P.A.Z. Suarez and J.C. Rubim, Anal. Chim. Acta 587 (2007) 194-199.

[34] R.M. Balabin and R.Z. Safieva, Fuel 87 (2008) 1096-1101.

[35] C. Lu, B. Xiang, G. Hao, J. Xu, Z. Wang and C. Chen, Journal of Near Infrared Spectroscopy 17 (2009) 59-67.

[36] P. Felizardo, P. Baptista, J.C. Menezes and M.J.N. Correia, Anal. Chim. Acta 595 (2007) 107-113.

[37] R.M. Balabin, R.Z. Safieva and E.I. Lomakina, Chemometr. Intell. Lab. 88 (2007) 183-188.

[38] R.M. Balabin, R.Z. Safieva and E.I. Lomakina, Chemometr. Intell. Lab. 93 (2008) 58-62.

[39] H. Yang, P.R. Griffiths and J.D. Tate, Anal. Chim. Acta 489 (2003) 125-136.

[40] Y. Li, C.W. Brown and S.-C. Lo, J. Near Infrared Spec. 7 (1999) 55-62.

[41] S. Sekulic, M.B. Seasholtz, Z. Wang and B.R. Kowalski, Anal. Chem. 65 (1993) 835-845.

[42] R.M. Balabin, Chem. Phys. 352 (2008) 267-275.

[43] R.M. Balabin, J. Chem. Phys. 129 (2008) 164101.

[44] R.M. Balabin, J. Chem. Phys. 131 (2009) 154307.

[45] R.M. Balabin, J. Phys. Chem. A 114 (2010) 3698-3702.

[46] A.G. Császár, W.D. Allen and H.F. Schaefer III, J. Chem. Phys. 108 (1998) 9751-9764. 
[47] R. M. Balabin, J. Chem. Phys. 132 (2010) 211103.

[48] G. Venkatasami, J. R. Sowa, Analytica Chimica Acta 665 (2010) 227-230.

[49] C.M. Bishop, Pattern Recognition and Machine Learning, Springer (2007).

[50] V.N. Vapnik, The Nature of Statistical Learning Theory, Springer-Verlag, New York (1995).

[51] S.R. Amendolia, G. Cossu, M.L. Ganadu, B. Golosio, G.L. Masala, G.M. Mura, Chemom. Intell. Lab. Syst. 69 (2003) 13-20.

[52] U. Thissen, M. Pepers, B. Ustun, W.J. Melssen, L.M.C. Buydens, Chemometr. Intell. Lab. Syst. 73 (2004) 169-179.

[53]F. Chauchard, R. Cogdill, S. Roussel, J.M. Roger, V. Bellon-Maurel, Chemometr. Intell. Lab. Syst. 71 (2004) 141-150.

[54] U. Thissen, Bulent Ustun, W.J. Melssen, L.M.C. Buydens, Anal. Chem. 76 (2004) 3099-3105.

[55] R. M. Balabin, J. Chem. Phys. 132 (2010) 231101.

[56] R. M. Balabin, Phys. Chem. Chem. Phys. 12 (2010) 5980.

[57] R.M. Balabin, "The First Step in Glycine Solvation: The Glycine-Water Complex" J. Phys. Chem. B (2011) in press. doi: 10.1021/jp107539z.

[58] X. Liu, G. Jia, C. Wu, K.g Wang and X. Wu, Journal of Near Infrared Spectroscopy 18 (2010) 113-120.

[59] U.S. Food and Drug Administration. Determination of melamine and cyanuric acid residues in infant formula using LC-MS/MS; http://www.cfsan.fda.gov/ frf/lib4421.htm.

[60] R. M. Balabin, E. I. Lomakina, J. Chem. Phys. 131 (2009) 074104.

[61] K. Ai, Y. Liu and L. Lu, J. Am. Chem. Soc. 131 (2009) 9496-9497. 